\title{
Asymptotic fluctuations and critical dimension for a two-level branching system
}

\author{
LUIS G. GOROSTIZA \\ Departamento de Matemáticas, Centro de Investigación y de Estudios Avanzados, A.P. 14-740, \\ 07000 Mexico, D.F., Mexico
}

The high-density asymptotic behaviour of a two-level branching system in $\mathbb{R}^{d}$ is studied. In the finitevariance case, a fluctuation limit process is obtained which is characterized as a generalized OrnsteinUhlenbeck process. In the case of critical branching at the two Jevels the long-time behaviour of the fluctuation limit process is shown to have critical dimension $2 \alpha$, where $\alpha$ is the index of the symmetric stable process representing the underlying particle motion. The same critical dimension has been obtained recently for the related (but qualitatively different) two-level superprocess. The fluctuation analysis uses different and simpler tools than the superprocess analysis.

Keywords: asymptotic fluctuations; critical dimension; two-level branching system

\section{Introduction}

Multilevel branching particle systems are mathematical models for a class of hierarchically structured populations. In such models the particles are grouped in subcollections that can disappear or be replicated at the different levels of the hierarchy. These models were introduced recently by Dawson and Hochberg (1991), and some of their properties and applications have been studied by Dawson et al. (1990; 1994; 1995a; 1995b), Dawson and Wu (1995), Etheridge (1993), Gorostiza et al. (1995), Hochberg (1995), and Wu (1991; $1993 ; 1994 a ; 1994 b)$. A different type of multilevel branching model has been considered by Greven (1991). Examples of multilevel branching systems which arise in computer science, molecular biology, population genetics and environmental science are mentioned in some of the references above. The analysis of multilevel branching models is complicated by the fact that the independence of behaviour in the particle dynamics which is present in the onelevel models no longer holds due to the higher-level branching.

An interesting feature of multilevel branching systems is the fact that in the case of critical branching the critical dimensions which separate two different long-time qualitative behaviours are of higher order than in the one-level models. For example, for the highdensity limit studied by Wu (199I; 1994a) and Gorostiza et al. (1995), known as the twolevel critical superprocess, the critical dimension for the persistence-extinction dichotomy

1350-7265 C 1996 Chapman \& Hall 
is 4 (see Remark 3.11), a result that was conjectured by Dawson, while it is 2 for the corresponding one-level model (see, for example, Gorostiza and Wakolbinger 1991). This result leads one to expect that the critical dimension 4 is an inherent property of the underlying two-level critical branching particle system, and therefore it should also appear in other ways. Some indication of this at the particle level appears in Hochberg and Wakolbinger (1995), and a result of related interest on clan dynamics was obtained by Stöckl and Wakolbinger (1994). In the present paper we show that 4 is also the critical dimension for the high-density fluctuation limit process (this result being a special case of Theorem 3.8).

While it is not surprising that the long-time behaviours of the two-level superprocess and the fluctuation limit process of the two-level system have the same critical dimension, it should be noted that the two processes are quite distinct in nature and that the techniques used to obtain them and to prove the corresponding critical dimension results are also different. One qualitative difference between the two processes is that in the critical, equilibrium, Brownian case the two-level superprocess is selfsimilar for $d=4$ (Wu 1994a), but the fluctuation limit process is not. Concerning the techniques, although the fluctuation analysis involves some difficult computations, the tools are simpler than those used for the superprocess analysis. Thus the fluctuation analysis provides a simpler way of finding the critical dimension for the long-time behaviour of the two-level branching system. Indeed, it reduces essentially to computing the limit of a variance. On the other hand, the superprocess contains all the information about the particle system (Dawson 1993). A natural question is whether the two critical dimension results can be derived from each other. This question, which by the way is aiso relevant to the one-level case, goes beyond the scope of this paper, but we comment on it in Remark 3.11.

A two-level superprocess is a Markov process with values in a space of measures, which is obtained as a limit of a two-level branching particle system under a high-density, shortlife, small-mass rescaling at the two levels simultaneously. Although this process is not the subject of this paper, we refer to it not only in connection with the question of the relationship between the critical dimensions raised in the previous paragraph, but also because it provides a motivation for the present study. The motivation is the following. The two-level superprocess can also be obtained in two steps, by first passing to the limit at the first level and then at the second level. The first-level limit yields a system of branching superprocesses, and it is this system which is our starting point in this paper. We note that in the two-step approach the two-level superprocess can be regarded as a 'super-superprocess', following the terminology of Dynkin (1991a), and that, as a consequence, the system of branching superprocesses is, at each time, a doubly stochastic Poisson random field whose random intensity is the two-level superprocess. This doubly stochastic Poisson relationship connects the critical long-time behaviours of the system of branching superprocesses and the two-level superprocess, and it shows that their persistence-extinction dichotornies are the same. This fact was exploited by Gorostiza et al. (1995) for the analysis of persistence-extinction of the critical two-level superprocess for which Wu $(1991 ; 1994 a)$ proved the extinction part.

Starting from the system of branching superprocesses that result from the first-level limit, 
our aim is to investigate the high-density hydrodynamic behaviour and the asymptotic fluctuations at the second level. Results of this type yield a qualitative insight into the collective behaviour of the two-level branching particle system which is different from that obtained by means of the two-level superprocess. To be precise, in this paper we begin this study for a particular two-level branching system in $\mathbb{R}^{d}$, and the process we investigate is the aggregated (or superposition) process of the two-level empirical measure process defined below. The aggregated process takes values in a space of tempered measures on $\mathbb{R}^{d}$, and therefore its fluctuation analysis can be carried out using $\mathcal{S}^{\prime}\left(\mathbb{R}^{d}\right)$, the space of tempered distributions on $\mathbb{R}^{d}$, as a state space. Finer results should be provided by the fluctuation analysis of the two-level empirical measure process, but this requires a nuclear space of distributions on a space of tempered measures on $\mathbb{R}^{d}$, which is not readily available. This problem is left for further study. However, although the aggregated process is coarser than the empirical measure process, it gives a considerable amount of information on the highdensity properties of the two-level branching system, in particular concerning the long-time behaviour.

A point worth emphasizing about the present study is the following. The $S^{\prime}\left(\mathbb{R}^{d}\right)$ valued processes that have been studied in most papers on fluctuation limits of particle systems concern the point measure-valued processes defined by the positions of particles that are randomly located in $\mathbb{R}^{d}$. In contrast, here we start from a random system of 'measure-valued particles', or 'superparticles', which are states of a collection of superprocesses.

Although some of the results below can also be proved for more general two-level branching particle systems, we restrict ourselves to a special model which is characterized by certain parameters, because we are interested in the dependence of the behaviour of the model on the parameter values, and in this way we can compare our results with others. Special cases of the present results (critical finite-variance branching and Brownian particle motion) appear without proof in Gorostiza (1995).

The paper is organized as follows. In Section 2 we define the two-level branching system we study and we recall some necessary background. Section 3 contains the results for the aggregated process: the law of large numbers (Theorem 3.1); fluctuation limit (Theorem 3.2 ; description of the fluctuation limit process as a generalized Ornstein-Uhlenbeck process (Theorem 3.4); and long-time behaviour of the fluctuation limit process in the critical case (Theorem 3.8). We determine the critical dimension in this case, and we comment on the relationship with the critical dimension of the related two-level superprocess (Remark 3.11). In addition, we note that in the case of equilibrium and supercritical dimensions, the high-density fluctuation limit and the long-time limit of the aggregated process are interchangeable (Theorem 3.10), and that the long-time limit of the fluctuation limit process has a representation in terms of the equilibrium state of a critical superprocess and the occupation time of an independent critical superprocess (Remark 3.12). In Section 4 we present the proofs. In Section 5 we mention some possible directions for further study. The Appendix contains the notation and the definitions of the spaces we use. A basic source on superprocesses is the lecture notes of Dawson (1993). Background on $\mathcal{S}^{\prime}\left(\mathbb{R}^{d}\right)$ can be found in Treves (1967), and on weak convergence of $\mathcal{S}^{\prime}\left(\mathbb{R}^{d}\right)$-valued processes in Mitoma (1983b). 


\section{A system of branching superprocesses}

For simplicity's sake we include all the parameters (even those which play a secondary role) in the name of the superprocess.

$\mathrm{A}(d, \alpha, \beta, V, b, c)$-superprocess $B=\{B(t), t \geq 0\}$ is a homogeneous Markov process with values in the space of measures $\mathcal{M}_{p}^{+}\left(\mathbb{R}^{d}\right)$ (see Appendix) whose characteristic functional is given by

$$
\mathrm{E}_{\mu}(\exp \{\mathrm{i}\langle B(t), \varphi\rangle\})=\exp \left\{\mathrm{i}\left\langle\mu, u_{\varphi}(t)\right\}\right\}, \quad t \geq 0, \mu \in \mathcal{M}_{p}^{+}\left(\mathbb{R}^{d}\right), \varphi \in \mathcal{S}\left(\mathbb{R}^{d}\right),
$$

where $\mathrm{E}_{\mu} \equiv \mathrm{E}[\cdot \mid B(0)=\mu], \alpha \in(0,2], \beta \in(0,1], V>0, b \in \mathbb{R}, c \in(0,1 /(1+\beta)], p$ is any real number such that $p>d / 2$ and in addition $p<(d+\alpha) / 2$ if $\alpha<2$, and $\left\{u_{\varphi}(x, t)\right.$, $\left.x \in \mathbb{R}^{d}, t \geq 0\right\}$ is a nonlinear semigroup which is the unique solution of the integral equation

$$
u(t)=e^{V b t} S_{i} \varphi-(-\mathrm{i})^{\beta} V_{c} \int_{0}^{t} \mathrm{e}^{V b(t-s)} S_{t-s}\left(u(s)^{1+\beta}\right) \mathrm{d} s,
$$

with $\left(S_{t}\right)$ designating the semigroup of the spherically symmetric $\alpha$-stable process in $\mathbb{R}^{d}$ (the case $\alpha=2$ corresponds to Brownian motion). The infinitesimal generator of $\left(S_{t}\right)$ is the fractional power of the Laplacian $\Delta_{\alpha} \equiv-(-\Delta)^{\alpha / 2}$. We denote the semigroup and the infinitesimal generator of the $(d, \alpha, \beta, V, b, c)$-superprocess by $\left(T_{t}\right)$ and $\mathcal{G}$, respectively. In the study of superprocesses it is more common to work with Laplace functionals and test functions in $C_{p}^{+}\left(\mathbb{R}^{d}\right)$. However, since the fluctuations of measure-valued processes are signed measure-valued and their limits are usually not signed measure-valued but they are $S^{\prime}\left(\mathbb{R}^{d}\right)$-valued, it is convenient to work with characteristic functionals and test functions in $\mathcal{S}\left(\mathbb{R}^{\alpha}\right)$ from the beginning.

We recall that the $(d, \alpha, \beta, V, b, c)$-superprocess characterized by $(2.1)$ and $(2,2)$ is obtained as a high-density, short-life, small-mass limit of a system of spherically symmetric $\alpha$-stable processes in $\mathbb{R}^{d}$, which in the $n$th rescaling consists of particles of mass $n^{-1}$, initially distributed with a density proportional to $n$, that branch at rate $V n^{\beta}$ with branching law generating function

$$
F(z)=z+b n^{-\beta}(z-1)+c(1-z)^{1+3}, \quad z \in \mathbb{C},|z|<1,
$$

(see, for example, Gorostiza and Lopez Mimbela 1993, or Dawson 1993, in the case $b=0$ ). Here $z^{1-\beta}=\exp \{(1+\beta) \log z\},-\pi / 2<\arg z<\pi / 2$, is the unique analytic continuation of $z^{1+\beta}$ for $z>0$.

In the critical branching case, which corresponds to $b=0$, the superprocess $B$ is persistent in dimensions $d>\alpha / \beta$ and it goes to local extinction as $t \rightarrow \infty$, if $d \leq \alpha / \beta$. Persistence means that if $B(0)=\lambda$, then $B(t) \Rightarrow B(\infty)$ in $\mathcal{M}_{p}^{+}\left(\mathbb{R}^{d}\right)$ as $t \rightarrow \infty$, where $B(\infty)$ has intensity measure $\lambda$. (Note that $\lambda$ is invariant for the semigroup $\left(S_{t}\right)$.) Moreover, $B(\infty)$ is an equilibrium state and an infinitely divisible random measure. Its canonical measure, which we denote by $R_{x}$, belongs to $\mathcal{M}_{p}^{2+}\left(\mathbb{R}^{d}\right.$ ) (a space of measures on $\mathcal{M}_{p}^{+}\left(\mathbb{R}^{d}\right)$, see Appendix), is invariant for the semigroup $\left(T_{t}\right)$, and has intensity measure $\lambda$ in the sense that

$$
\left\langle\left\langle R_{\infty},\langle\cdot, \varphi\rangle\right\rangle\right\rangle=\langle\lambda, \varphi\rangle \text {. }
$$


(For these results, see Dawson 1993; Dawson and Perkins 1991; Gorostiza and Wakolbinger 1991; Wu 1991; 1994a.)

$\mathrm{A}\left(\beta_{2}, V_{2}, b_{2}, c_{2}\right)$-branching $\left(d, \alpha, \beta_{1}, V_{1}, b_{1}, c_{1}\right)$-superprocess is a spatial branching system in $\mathcal{M}_{p}^{+}\left(\mathbb{R}^{d}\right)$. Its measure-valued particles, which we call 'superparticles' for short, migrate according to a $\left(d, \alpha, \beta_{1}, V_{1}, b_{1}, c_{1}\right)$-superprocess and branch at rate $V_{2}$ with branching law generating function

$$
G(z)=z+b_{2}(z-1)+c_{2}(1-z)^{1+\beta_{2}}, \quad z \in \mathbb{C},|z|<1,
$$

where $\beta_{2} \in(0,1], b_{2} \in\left(-1, c_{2}\right], c_{2} \in\left(0,\left(1+b_{2}\right) /\left(1+\beta_{2}\right)\right]$. The offspring superparticles start off from the branching site of their parent, and the motions, lifetimes and branchings of different superparticles are independent of each other.

We consider a system of $\left(\beta_{2}, V_{2}, b_{2}, c_{2}\right)$-branching $\left(d, \alpha, \beta_{1}, V_{1}, b_{1}, c_{1}\right)$-superprocesses with initial configuration given by a realization of a Poisson random measure on $\mathcal{M}_{p}^{+}\left(\mathbb{R}^{d}\right)$ with intensity measure $R \in \mathcal{M}_{p}^{2+}\left(\mathbb{R}^{d}\right)$, and such that their evolutions are independent of each other given the initial configuration. We denote by $B_{i j}(t)$ the location in $\mathcal{M}_{p}^{+}\left(\mathbb{R}^{d}\right)$ at time $t$ of the $j$ th superparticle in the $i$ th branching superprocess.

The empirical measure process $Y \equiv\{Y(t), t \geq 0\}$ of the system is defined by

$$
Y(t)=\sum_{i} \sum_{j} \delta_{B_{i j}(t)}
$$

Then $Y$ is an $\mathcal{M}_{p}^{2+}\left(\mathbb{R}^{d}\right)$-valued Markov process, and its high-density, short-life, small-mass limit (i.e. superprocess limit) coincides with the two-level superprocess studied by $\mathrm{Wu}$ $(1991 ; 1993 ; 1994 a)$ (in the critical branching case and $\alpha=2$ ). As stated in the Introduction, the fluctuation analysis of the process $Y$ requires a nuclear space of distributions on $\mathcal{M}_{p}^{+}\left(\mathbb{R}^{d}\right)$. A methodology for such an analysis has not yet been developed, and therefore we will study instead the fluctuations of the corresponding aggregated process.

The aggregated process $\bar{Y} \equiv\{\bar{Y}(t), t \geq 0\}$ of $Y$ (or of the system) is given by

$$
\bar{Y}(t)=\sum_{i} X_{i}(t), \quad \text { where } X_{i}(t)=\sum_{j} B_{i j}(t) .
$$

The process $\bar{Y}$ is the aggregated process of $Y$ in the sense that the masses $B_{i j}(t)$ are not distinguished, but summed. Note from (2.4) and (2.5) that $\langle\langle Y(t),\langle\cdot, \varphi\rangle\rangle\rangle=\langle\bar{Y}(t), \varphi\rangle$, $\varphi \in \mathcal{S}\left(\mathbb{R}^{d}\right)$.

We observe that $\bar{Y}$ may be assumed to take values in $\mathcal{M}_{p}^{+}\left(\mathbb{R}^{d}\right)$ because each $X_{i}$ is $\mathcal{M}_{p}^{+}\left(\mathbb{R}^{d}\right)$-valued and $\bar{Y}(0)$ has locally finite intensity, $\bar{Y}$ is not Markovian (even though $Y$ is Markovian), the conditional characteristic functional of $\bar{Y}(t)$ with respect to the superparticle system $Y(s)$ is given by

$$
\begin{aligned}
\mathrm{E}[\exp & \{\mathrm{i}\langle\bar{Y}(t), \varphi)\} \mid Y(s)] \\
& =\exp \{\langle(Y(s), \log \mathrm{E}(\exp \{\mathrm{i}\langle X(t-s), \varphi\rangle\})\}\rangle\}, \quad s \leq t, \varphi \in \mathcal{S}\left(\mathbb{R}^{d}\right),
\end{aligned}
$$

and $\bar{Y}$ has finite second moments if and only if $\beta_{1}=\beta_{2}=1$.

We wish to study the high-density behaviour of the process $\bar{Y}$. Since the fluctuations of $\bar{Y}$ 
take values in $\mathcal{M}_{p}\left(\mathbb{R}^{d}\right)$, and $\mathcal{M}_{p}\left(\mathbb{R}^{d}\right) \subset \mathcal{S}^{\prime}\left(\mathbb{R}^{d}\right)$, we may use available methods for the analysis of $S^{\prime}\left(\mathbb{R}^{d}\right)$-valued processes.

\section{High-density limits}

For all $n=1,2, \ldots$, let $\bar{Y}^{n} \equiv\left\{\bar{Y}^{n}(t), t \geq 0\right\}$ be the aggregated process of the system of $\left(\beta_{2}, V_{2}, b_{2}, c_{2}\right)$-branching $\left(d, \alpha, \beta_{1}, V_{1}, b_{1}, c_{1}\right)$-superprocesses described in Section 2, with initial configuration given by a realization of a Poisson random measure on $\mathcal{M}_{p}^{+}\left(\mathbb{R}^{d}\right)$ with intensity measure $n R$, where $R \in \mathcal{M}_{p}^{2+}\left(\mathbb{R}^{d}\right)$.

Theorem 3.1 (Law of large numbers). For all $t \geq 0$ and $\varphi \in \mathcal{S}\left(\mathbb{R}^{d}\right)$,

$$
n^{-1}\left\langle\bar{Y}^{n}(t), \varphi\right\rangle \rightarrow\left\langle\left\langle R,\left\langle\cdot, S_{t} \varphi\right\rangle\right\rangle \mathrm{e}^{\left(V_{1} b_{1}+V_{2} b_{2}\right) t} \quad \text { in probability as } n \rightarrow \infty .\right.
$$

In the case $\beta_{1}=\beta_{2}=1$ the convergence also holds in mean square.

For the fluctuation results we assume finite second-moment branching at both levels $\left(\beta_{1}=\beta_{2}=1\right)$. The fluctuation process $Z^{n} \equiv\left\{Z^{n}(t), t \geq 0\right\}$ of $\bar{Y}^{n}$ is defined by

$$
Z^{n}(t)=n^{-1 / 2}\left(\bar{Y}^{n}(t)-\mathrm{E} \bar{Y}^{n}(t)\right) .
$$

Theorem 3.2 (Fluctuation limit). The finite-dimensional distributions of the process $Z^{n}$ converge weakly as $n \rightarrow \infty$ to those of the $\mathcal{S}^{\prime}\left(\mathbb{R}^{d}\right)$-valued centred Gaussian process $Z \equiv$ $\{Z(t), t \geq 0\}$ with covariance functional given by

$$
\begin{aligned}
\operatorname{cov}(\langle Z(s), \varphi\rangle,\langle Z(t), \psi\rangle)= & \operatorname{cov}\left(\langle Z(s), \varphi\rangle,\left\langle Z(s), \mathrm{e}^{\left(V_{1} b_{1}+V_{2} b_{2}\right)(t-s)} S_{t-s} \psi\right\rangle\right), \\
& s \leq t, \varphi, \psi \in \mathcal{S}\left(\mathbb{R}^{d}\right),
\end{aligned}
$$

where

$$
\begin{aligned}
& \operatorname{cov}(\langle Z(s), \varphi\rangle,\langle Z(s\rangle, \psi\rangle) \\
&=\mathrm{e}^{2\left\langle V_{1} b_{1}+V_{2} b_{2}\right) s}\left\{\left[1+\frac{2 c_{2}-b_{2}}{b_{2}}\left(1-\mathrm{e}^{-V_{2} b_{2} s}\right]\left\langle\left\langle R,\left\langle\cdot, S_{s} \varphi\right\rangle\left\langle\cdot, S_{s} \psi\right\rangle\right\rangle\right\rangle\right.\right. \\
&+2 V_{1} c_{1} \int_{0}^{s} \mathrm{e}^{-\left(V_{1} b_{1}+V_{2} b_{2}\right) r}\left\langle\left\langle R,\left\langle\cdot, S_{r}\left(\left(S_{s-r} \varphi\right)\left(S_{s-r} \psi\right)\right)\right\rangle\right\rangle\right\rangle \mathrm{d} r \\
&+2 V_{1} c_{1} V_{2}\left(2 c_{2}-b_{2}\right) \int_{0}^{s} \mathrm{e}^{-\left(V_{1} b_{1}+V_{2} b_{2}\right) r} \\
&\left.\times \int_{0}^{r} \mathrm{e}^{V_{1} b_{1}{ }^{*}}\left\langle\left\langle R_{r}\left(\cdot, S_{r-v}\left(\left(S_{s-r+v} \varphi\right)\left(S_{s-r+v} \psi\right)\right)\right\rangle\right\rangle\right\rangle \mathrm{d} v \mathrm{~d} r\right\}
\end{aligned}
$$

(the case $b_{2}=0$ is included).

Remark 3.3. It is possible to prove weak convergence in the Skorokhod space $D([0, \infty)$, $\mathcal{S}^{\prime}\left(\mathbb{R}^{d}\right)$ ) by standard methods. The tightness proof involves unpleasant calculations. Since 
the limit $Z$ has continuous paths, weak convergence in $D\left([0, \infty), \mathcal{S}^{\prime}\left(\mathbb{R}^{d}\right)\right)$ can be proved in a simpler way by a method described by Fernández and Gorostiza (1992), which is based on results due to Aldous (1989) and Cremers and Kadelka (1986).

Theorem 3.4 ( $Z$ as a generalized Ornstein-Uhlenbeck process). The fluctuation limit process $Z$ is a Markov process with continuous paths, and it is an Ornstein-Uhlenbeck process governed by the Langevin equation

$$
\mathrm{d} Z(t)=\left(\Delta_{\alpha}^{*}+V_{1} b_{1}+V_{2} b_{2}\right) Z(t) \mathrm{d} t+\mathrm{d} W(t), \quad t>0,
$$

and with $Z(0)$ an $S^{\prime}\left(\mathbb{R}^{d}\right)$-valued centred Gaussian random variable with covariance functional

$$
\operatorname{cov}(\langle Z(0), \varphi\rangle,\langle Z(0), \psi\rangle)=\langle\langle R,\langle\cdot, \varphi\rangle\langle\cdot, \psi\rangle\rangle\rangle,
$$

where $W$ is an inhomogeneous $\mathcal{S}^{\prime}\left(\mathbb{R}^{d}\right)$-Wiener process with covariance functional

$$
\operatorname{cov}(\langle W(s), \varphi\rangle,\langle W(t), \psi\rangle)=\int_{0}^{s \wedge t} Q_{u}(\varphi, \psi) \mathrm{d} u
$$

with

$$
\begin{aligned}
Q_{u}(\varphi, \psi)= & \mathrm{e}^{\left(V_{1} b_{1}+V_{2} b_{2}\right) u}\left\{2 V_{1} c_{1}\left\langle\left\langle R,\left\langle\cdot, S_{u}(\varphi \psi)\right\rangle\right\rangle\right\rangle+V_{2}\left(2 c_{2}-b_{2}\right)\left\langle\left\langle R_{,}\left\langle\cdot, S_{u} \varphi\right\rangle\left\langle\cdot, S_{u} \psi\right\rangle\right\rangle\right\rangle\right. \\
& \left.+2 V_{1} c_{1} V_{2}\left(2 c_{2}-b_{2}\right) \int_{0}^{u} \mathrm{e}^{V_{1} b_{1} v}\left\langle\left\langle R,\left\langle\cdot, S_{u-v}\left(\left(S_{v} \varphi\right)\left(S_{v} \psi\right)\right)\right\rangle\right\rangle\right\rangle \mathrm{d} v\right\}
\end{aligned}
$$

Remark 3.5. The generalized Langevin equation above is understood in a weak sense, i.e. tested on functions $\varphi \in \mathcal{S}\left(\mathbb{R}^{d}\right)$ and integrated in $t$. Moreover, in the case $\alpha<2$ the solution to the equation is interpreted in the extended sense introduced by Dawson and Gorostiza $(1990)$, because $\Delta_{o}^{*}$ does not map $\mathcal{S}^{\prime}\left(\mathbb{R}^{d}\right)$ into itself. (For background on $\mathcal{S}^{\prime}$-Wiener processes and $\mathcal{S}^{\prime}$-Langevin equations, see Bojdecki and Gorostiza 1986; Bojdecki and Jakubowski 1990; Dawson and Gorostiza 1989; 1990).

Remark 3.6. If the initial intensity measure $R$ is given by $R=\delta_{\delta_{x}} \lambda(\mathrm{d} x)$ (which belongs to $\mathcal{M}_{p}^{2+}\left(\mathbb{R}^{d}\right)$; see Wu 1991), then $Z(0)$ is the standard Gaussian white noise measure on $\mathcal{S}^{\prime}\left(\mathbb{R}^{d}\right)$.

Remark 3.7. The (one-level) high-density fluctuation limit of a system of branching processes with characteristics $\alpha, \beta, V_{1}, b_{1}, c_{1}$ (and initial Poisson random measure with intensity $\lambda$ ) is a generalized Ornstein-Uhlenbeck process $Z$ with Langevin equation

$$
\mathrm{d} Z(t)=\left(\Delta_{\alpha}^{*}+V_{1} b_{1}\right) Z(t) \mathrm{d} t+\mathrm{d} W(t),
$$

where $W$ is the $S\left(\mathbb{R}^{d}\right)$-Wiener process with covariance kernel

$$
Q_{u}(\varphi, \psi)=\mathrm{e}^{V_{1} b_{1} u}\left\langle\lambda, V_{1}\left(2 c_{1}-b_{1}\right) \varphi \psi-\varphi \Delta_{\alpha} \psi-\psi \Delta_{\alpha} \varphi\right\rangle .
$$

(this is a special case of the result in Fernández and Gorostiza 1990). The terms in $Q_{u}$ involving $\Delta_{\alpha}$ represent the effect in the randomness of the limit $Z$ caused by the particle 
motion. The lack of similar terms in $Q_{u}$ in the two-level case (Theorem 3.4) is due to the aggregation.

The next result gives the long-time behaviour of the process $Z$ in the case of critical branching at the two levels.

Theorem 3.8 (Long-time behaviour of $Z$ in the critical case). Let $b_{1}=b_{2}=0$, and $d>2 \alpha$. If $R=R_{\infty}$ or $R=\delta_{\delta_{x}} \lambda(\mathrm{d} x)$, then $Z(t) \Rightarrow Z(\infty)$ as $t \rightarrow \infty$, where $Z(\infty)$ is an $S^{\prime}\left(\mathbb{R}^{d}\right)$-valued centred Gaussian random variable with covariance functional given by

$$
\operatorname{cov}(\langle Z(\infty), \varphi\rangle,\langle Z(\infty), \psi\rangle)=\int_{\mathbb{R}^{d}} \int_{\mathbb{R}^{d}} \varphi(x) \psi(y)\left[k_{\mathrm{I}}(x, y)+k_{2}(x, y)\right] \mathrm{d} x \mathrm{~d} y,
$$

where

$$
k_{1}(x, y)=\frac{V_{1} c_{1} \Gamma\left(\frac{d-\alpha}{2}\right)}{2^{\alpha} \pi^{d / 2} \Gamma\left(\frac{\alpha}{2}\right)} \frac{1}{\|x-y\|^{d-\alpha}}
$$

and

$$
k_{2}(x, y)=\frac{V_{1} c_{1} V_{2} c_{2} \Gamma\left(\frac{d-2 \alpha}{2}\right) \Gamma\left(\frac{\alpha}{2}\right)}{4^{\alpha} \pi^{(d-2) / 2} \Gamma\left(\frac{\alpha+1}{2}\right)} \frac{1}{\|x-y\|^{d-2 \alpha}} .
$$

for $d \leq 2 \alpha, Z(t)$ does not converge as $t \rightarrow \infty$.

Remark 3.9. Under the hypotheses of Theorem 3.8, the covariance kernel $Q_{t}$ of the $\mathcal{S}^{\prime}\left(\mathbb{R}^{d}\right)$ Wiener process in Theorem 3.4 has the following limiting behaviour:

$$
Q_{t}(\varphi, \psi)=2 V_{1} c_{1}\langle\lambda, \varphi \psi\rangle+R_{t}(\varphi, \psi)
$$

and

$$
\lim _{t \rightarrow \infty} R_{t}(\varphi, \psi)=\frac{V_{1} c_{1} V_{2} c_{2} \Gamma\left(\frac{d-\alpha}{2}\right)}{2^{\alpha-1} \pi^{d / 2} \Gamma\left(\frac{\alpha}{2}\right)} \int_{\mathbf{R}^{d}} \int_{\mathbf{R}^{d}} \frac{\varphi(x) \psi(y)}{\|x-y\|^{d-\alpha}} \mathrm{d} x \mathrm{~d} y .
$$

(This can be shown by the same arguments in the proof of Theorem 3.8.) Note that in the limit the two terms correspond respectively to those in the covariance functional of $Z(\infty)$ in Theorem 3.8. The first term, which represents a spatial white noise, is the same as in the onelevel case (Remark 3.7), and the second term involves the combined effects from the two levels.

We now give an interchange of limits result in the case of critical branching and $d>2 \alpha$. Starting from a Poisson random field with intensity measure $R_{\infty}$, we have $\bar{Y}(t) \Rightarrow \bar{Y}(\infty)$ 
as $t \rightarrow \infty$, where $\bar{Y}(\infty)$ is an $\mathcal{S}^{\prime}\left(\mathbb{R}^{d}\right)$-random variable with characteristic functional given by

$$
\mathrm{E}(\exp \{\mathrm{i}\langle\bar{Y}(\infty), \varphi\rangle\})=\exp \left\{-\left\langle\left\langle R_{\infty}, 1-\mathrm{e}^{\mathrm{i}\langle\cdot \varphi \varphi\rangle}\right\rangle\right\rangle+V_{2} c_{2} \int_{0}^{\infty}\left\langle\left\langle R_{\infty}, w_{\varphi}(t)^{2}\right\rangle\right\rangle \mathrm{d} t\right\},
$$

and $w_{\varphi}(\mu, t)$ is defined by (4.11) below. (Weak convergence is proved by means of the Laplace functional, and the passage from the Laplace functional to the characteristic functional is done by analytic continuation, as in Iscoe 1986.) Let $\bar{Y}^{n}(\infty)$ denote the limit above corresponding to the initial Poisson intensity measure $n R_{\infty}$, and consider the fluctuation random field $H^{n}$ defined by

$$
H^{n}=n^{-1 / 2}\left(\bar{Y}^{n}(\infty)-\mathrm{E}(\bar{Y} n(\infty))\right) \text {. }
$$

Theorem 3.10 (Interchange of limits $t \rightarrow \infty$ and $n \rightarrow \infty$ ). Let $d>2 \alpha$. Then

$$
H^{n} \Rightarrow Z(\infty) \quad \text { as } n \rightarrow \infty,
$$

where $Z(\infty)$ is the long-time limit of the fuctuation limit process $Z$ described in Theorem 3.8 .

Remark 3.11. Theorem 3.8 shows that $2 \alpha$ is the critical dimension for the long-time behaviour of the fluctuation limit process $Z$. For the two-level superprocess constructed from the same critical two-level branching particle system in the case $\alpha=2$, Wu (1991; 1994a) proved that local extinction occurs in dimensions $d \leq 4$ if the initial state is $R_{\infty}$ or $\delta_{\delta_{x}} \lambda(\mathrm{d} x)$ (for the precise meaning of local extinction of the two-level superprocess, see $\mathrm{Wu}$ $1991 ; 1994 a)$, and from his results in Wu (1991) it follows that there is no extinction in dimensions $d>4$ (because the second-moment measure of the aggregated process of the two-level superprocess has a non-zero limit as $t \rightarrow \infty$ ). For the same two-level superprocess, Gorostiza et al. (1995) proved persistence in dimensions $d>4$ if the initial state is $R_{\infty}$. Hence 4 is the critical dimension for the critical two-level superprocess. These two-level superprocess persistence-extinction results should also hold for $\alpha<2$ and the critical dimension should be $2 \alpha$ (with similar proofs to those for the case $\alpha=2$ ). The critical dimensions for the two-level superprocess and for the fluctuation limit process $Z$ have been obtained independently of each other. Clearly, they must be related since they arise from the same two-level branching particle system. It is natural to ask if they can be derived from each other. The same question is relevant for the one-level case, where the critical dimension is $\alpha$. In this case one can show, using the doubly stochastic Poisson relationship and an interchange of the $t \rightarrow \infty$ and $n \rightarrow \infty$ limits, that persistence of the superprocess for $d>\alpha$ implies existence of the long-time limit of the fluctuation limit process, and this long-time limit can be identified this way (the limit covariance appears in Gorostiza 1983, for $\alpha=2$ ). An analogous procedure could be foliowed in the two-level case for $d>2 \alpha$ in order to prove existence of, and identify, the long-time limit of the fluctuation limit process for the empirical measure process (and hence for the aggregated process), but this requires the use of Lévy's continuity theorem, for which a nuclear space of distributions on $\mathcal{M}_{p}\left(\mathbb{R}^{d}\right)$ (which we do not have) is necessary. In any case, for both the one-level and two-level 
models, in supercritical dimensions the persistence of the superprocess implies the long-time behaviour of the fluctuation limit process. But using superprocess persistence results to prove long-time fluctuation results means using a more difficult class of results to prove something which can be done directly more easily. A more interesting and non-trivial question is whether long-time fluctuation limit results can be used to prove persistence of superprocesses.

Remark 3.12. (A representation of $Z(\infty)$ ). Let $d>2 \alpha$. From the form of the covariance functional of $Z(\infty)$ in Theorem 3.8 it follows that $Z(\infty)$ is equal in distribution to $Z_{1}+Z_{2}$, where $Z_{1}$ and $Z_{2}$ are independent random fields, $Z_{1}$ is the high-density fluctuation limit as $n \rightarrow \infty$ of the equilibrium state of a critical superprocess with intensity $n \lambda$, and $Z_{2}$ is the long-time fluctuation limit of the occupation time of a critical superprocess with intensity $\lambda$ (see Iscoe 1986, Theorem 5.5, for the second part of this representation). Thus, $\bar{Y}^{n}(t)$ is distributed approximately as

$$
\left(V_{1} c_{1}\right)^{1 / 2} B_{1}^{n}(\infty)+\left(V_{1} c_{1} V_{2} c_{2}\right)^{1 / 2} t^{-1 / 2}\left[\int_{0}^{t} B_{2}(s) d s-\lambda t\right],
$$

for large $n$ and $t$, where $B_{1}^{n}(\infty)$ is the equilibrium state of a (critical) $(d, \alpha, 1,2,0,1 / 2)$ superprocess $B_{1}^{n}$ with intensity $n \lambda$, and $B_{2}$ is an independent (critical) $(d, \alpha, 1,2,0,1 / 2)$ superprocess with intensity $\lambda$. What is of interest in this representation is that the asymptotic behaviour of the two-level process $\bar{Y}^{n}(t)$ is expressed in terms of the onelevel processes $B_{1}^{n}$ and $B_{2}$. This leads one to believe that there is a relationship between the two-level system and the occupation time of the one-level system.

Remark 3.13. In Theorems 3.8 and 3.10 the Poisson intensity $R_{\infty}$ has an associated branching rate (the parameter $V_{1}$ in the $\left(d, \alpha_{1}, \beta_{1}, V_{1}, 0, c_{1}\right)$-superprocess; see Lemma 4.6 below). However, we can choose any measure for the Poisson intensity $R$. In particular, we may let $R$ be the canonical measure of another (critical, persistent) superprocess; for example, we can take a different value of $V_{1}$ for $R_{\infty}$. Having noted this point, special cases of the previous results with no branching at the first or second levels can be obtained by setting the branching rates $V_{1}$ and/or $V_{2}$ equal to $0: V_{1}=V_{2}=0$ (system of deterministic $S_{1}$-evolutions); $V_{1}=0$ and $V_{2}>0$ (system of branching deterministic $S_{t}$-evolutions); $V_{1}>0$ and $V_{2}=0$ (system of superprocesses). This observation is also relevant in connection with Example 3.4.2 in Bojdecki and Gorostiza (1996).

\section{Proofs}

Lemma 4.1. Let $X \equiv\{X(t), t \geq 0\}$ be the $\mathcal{M}_{p}^{+}\left(\mathbb{R}^{d}\right)$-valued process defined by $X(t)=\sum_{j} B_{j}(t)$, where $B_{j}(t)$ is the location at time $t$ of the $j$ th superparticle in the $\left(\beta_{2}, V_{2}, b_{2}, c_{2}\right)$-branching $\left(d, \alpha, \beta_{1}, V_{1}, b_{1}, c_{1}\right)$-superprocess. Then

$$
\mathrm{E}_{\mu}(\langle X(t), \varphi\rangle)=\left\langle\mu, S_{t} \varphi\right\rangle \mathrm{e}^{\left(V_{1} b_{1}+V_{2} b_{2}\right) t},
$$


and, if $\beta_{1}=\beta_{2}=1$,

$$
\begin{aligned}
\mathrm{E}_{\mu}\left(\langle X(t), \varphi\rangle^{2}\right)= & \mathrm{e}^{2\left(V_{1} b_{1}+V_{2} b_{2}\right) r}\left\{\left[1+\frac{2 c_{2}-b_{2}}{b_{2}}\left(1-\mathrm{e}^{-V_{2} b_{2} t}\right)\right]\left\langle\mu, S_{t} \varphi\right\rangle^{2}\right. \\
& +2 V_{1} c_{1} \int_{0}^{t} \mathrm{e}^{-\left(V_{1} b_{1}+V_{2} b_{2}\right) r}\left(\mu, S_{r}\left(S_{t-r} \varphi\right)^{2}\right\rangle \mathrm{d} r \\
& \left.+2 V_{1} c_{1} V_{2}\left(2 c_{2}-b_{2}\right) \int_{0}^{t} \mathrm{e}^{-\left(V_{1} b_{1}+V_{2} b_{2}\right) r} \int_{0}^{r} \mathrm{e}^{V_{1} b_{1} v}\left\langle\mu, S_{r-v}\left(S_{t-r+v} \varphi\right)^{2}\right\rangle \mathrm{d} v \mathrm{~d} r\right\}
\end{aligned}
$$

(the case $b_{2}=0$ is included), and

$$
\mathrm{E}_{\mu}(\langle X(s), \varphi\rangle\langle X(t), \psi\rangle)=\mathrm{E}_{\mu}\left(\langle X(s), \varphi\rangle\left\langle X(s), S_{t-s} \psi\right\rangle\right) \mathrm{e}^{\left(V_{1} b_{1}+V_{2} b_{2}\right)(t-s)}, \quad s \leq t
$$

Proof. We abbreviate the proof by referring to analogous calculations in previous papers.

Computing as in Gorostiza and Roelly (1991, proof of Theorem 3.5) we have, recalling that $\left(T_{t}\right)$ is the semigroup of the superprocess $B$,

$$
\mathrm{E}_{\mu}(\langle B(t), \varphi\rangle)=T_{t}(\langle\cdot, \varphi\rangle)(\mu)=\left\langle\mu, S_{t} \varphi\right\rangle \mathrm{e}^{V_{1} b_{1} t}
$$

and

$$
\begin{aligned}
\mathrm{E}_{\mu}\left((B(t), \varphi\rangle^{2}\right) & =T_{t}\left(\langle\cdot, \varphi\rangle^{2}\right)(\mu) \\
& =\left\langle\mu, S_{t} \varphi\right\rangle^{2} \mathrm{e}^{2 V_{1} b_{1} t}+2 V_{1} c_{1} \int_{0}^{t} \mathrm{e}^{V_{1} b_{1}(t+s)}\left\langle\mu, S_{t-s}\left(S_{s} \varphi\right)^{2}\right\rangle \mathrm{d} s
\end{aligned}
$$

and, noting that $X(t)$ is the aggregated process of $\sum_{j} \delta_{B_{i}(t)}$ and calculating as in Fernández and Gorostiza (1990, proof of Lemma 1), we have

$$
\mathrm{E}_{\mu}(\langle X(t), \varphi\rangle)=T_{t}(\langle\cdot, \varphi\rangle)(\mu) \mathrm{e}^{V_{2} b_{2} t}
$$

and

$$
\begin{aligned}
\mathrm{E}_{\mu}\left((X(t), \varphi\rangle^{2}\right)= & \left(T_{t}(\langle\cdot, \varphi\rangle)(\mu)\right)^{2} \mathrm{e}^{2 V_{2} b_{2} t} \\
& +\mathrm{e}^{2 V_{2} b_{2} t} \int_{0}^{t} \mathrm{e}^{-V_{2} b_{2} r} T_{r}\left[\mathcal{G}\left(\left(T_{t-r}(\langle\cdot, \varphi\rangle)\right)^{2}\right)\right. \\
& -2\left(T_{t-r}(\langle\cdot, \varphi))\right) \mathcal{G}\left(T_{t-r}(\langle\cdot, \varphi\rangle)\right) \\
& \left.+V_{2}\left(2 c_{2}-b_{2}\right)\left(T_{t-r}(\langle\cdot, \varphi\rangle)\right)^{2}\right](\mu) \mathrm{d} r .
\end{aligned}
$$

It is easy to verify that the generator $\mathcal{G}$ of the superprocess $B$ is given as follows for the functions $F(\mu)=\langle\mu, \varphi\rangle$ and $F(\mu)=\langle\mu, \varphi\rangle^{2}$ :

and

$$
\mathcal{G}(\langle\cdot, \varphi\rangle)(\mu)=\left\langle\mu,\left(\Delta_{\alpha}+V_{1} b_{1}\right) \varphi\right\rangle
$$

$$
\mathcal{G}\left(\langle\cdot, \varphi\rangle^{2}\right)(\mu)=2\langle\mu, \varphi\rangle\left\langle\mu,\left(\Delta_{\alpha}+V_{1} b_{1}\right) \varphi\right\rangle+2 V_{1} c_{1}\left\langle\mu, \varphi^{2}\right\rangle
$$

From (4.4) and (4.6) we obtain (4.1), and from (4.4)-(4.9) we obtain (4.2). 
Finally, (4.3) follows from (4.1), conditioning on $\{X(r), r \leq s\}$, and the Markov property of $X$ with respect to the filtration generated by $\sum_{j} \delta_{B_{j}(t)}$.

Corollary 4.2. Let $\bar{Y}$ be the aggregated process of the system of $\left(\beta_{2}, V_{2}, b_{2}, c_{2}\right)$-branching $\left(d, \alpha, \beta_{1}, V_{1}, b_{1}, c_{1}\right)$-superprocesses with initial Poisson measure with intensity $R \in \mathcal{M}_{p}^{2+}\left(\mathbb{R}^{d}\right)$. Then

$$
E\langle\bar{Y}(t), \varphi\rangle=\left\langle\left\langle R,\left\langle\cdot, S_{t} \varphi\right)\right\rangle\right\} \mathrm{e}^{\left(V_{1} b_{1}+V_{2} b_{2}\right) t}
$$

Proof. Since $\mathrm{E}\langle\bar{Y}(t), \varphi\rangle=\langle\langle R, E .(\langle X(t), \varphi\rangle)\rangle\rangle$, the result follows from (4.1).

The previous results are enough to prove the law of large numbers in the finite secondmoment case and the fluctuation limit of the one-dimensional distributions.

Proof of Theorem 3.1 (with $\beta_{1}=\beta_{2}=1$ ). From the assumption that $Y^{n}(0)$ is Poisson with intensity $n R$ and (4.10) we have

$$
\begin{aligned}
& \mathrm{E}\left|n^{-1}\left\langle\bar{Y}^{n}(t), \varphi\right\rangle-\left\langle\left\langle R,\left\langle\cdot, S_{t} \varphi\right\rangle\right\rangle\right\rangle \mathrm{e}^{\left(V_{1} b_{1}+V_{2} b_{2}\right) t}\right|^{2} \\
& \quad=n^{-1}\left\langle\left\langle R, \mathrm{E} \cdot\left(\langle X(t), \varphi\rangle^{2}\right)\right\rangle\right\rangle \rightarrow 0 \quad \text { as } n \rightarrow \infty,
\end{aligned}
$$

since the assumption on $R$ and (4.2) assure the finiteness of $\left\langle\left\langle R, \mathrm{E} .\left(\langle X(t), \varphi\rangle^{2}\right)\right\rangle\right\rangle$.

Lemma 4.3. For all $t \geq 0$ and all $\varphi \in \mathcal{S}\left(\mathbb{R}^{d}\right)$.

$$
\lim _{n \rightarrow \infty} \mathrm{E}\left(\exp \left\{\mathrm{i}\left\langle Z_{n}(t), \varphi\right\rangle\right\}\right)=\exp \left\{-\frac{1}{2}\left\langle\left\langle R, \mathrm{E} .\left(\langle X(t), \varphi\rangle^{2}\right)\right\}\right\rangle\right\}
$$

and therefore $Z_{n}(t) \Rightarrow Z(t)$ as $n \rightarrow \infty$.

Proof. Since $Y^{n}(0)$ is Poisson with intensity $n R$ we have, from (3.1),

$$
\mathrm{E}\left(\exp \left\{\mathrm{i}\left\langle Z_{n}(t), \varphi\right\rangle\right\}\right)=\exp \left\{-\mathrm{i} n^{-1 / 2} \mathrm{E}\left\langle\bar{Y}_{n}(t), \varphi\right\rangle+\left\langle\left\langle n R, \mathrm{E} \cdot\left(\exp \left\{\mathrm{in}^{-1 / 2}\langle X(t), \varphi\rangle\right\}\right)-1\right\rangle\right\rangle\right\} .
$$

Expanding the exponential in the second term on the right-hand side and using $\mathrm{E}\left(\left\langle\bar{Y}_{n}(t), \varphi\right\rangle\right)=\langle\langle n R, \mathrm{E} .(\langle X(t), \varphi\rangle)\rangle\rangle$ and $\left\langle\left\langle R, \mathrm{E} .\left(\langle X(t),|\varphi|\rangle^{3}\right)\right\rangle\right\rangle<\infty$ (which holds because $\left.\beta_{\mathrm{I}}=\beta_{2}=2\right)$, we have

$$
\mathrm{E}\left(\exp \left\{\mathrm{i}\left\langle Z_{n}(t), \varphi\right\rangle\right\}\right)=\exp \left\{-\frac{1}{2}\left\langle\left(R, \mathrm{E} \cdot\left(\langle X(t), \varphi\rangle^{2}\right)\right\rangle\right\rangle+o(1)\right\} \quad \text { as } n \rightarrow \infty,
$$

which by (4.2) gives the desired result.

In order to proceed further we must work with the nonlinear structure of the system. Let

$$
w_{\varphi}(\mu, t)=1-\mathrm{E}_{\mu}(\exp \{\mathbf{i}\langle X(t), \varphi\rangle\}), \quad \mu \in \mathcal{M}_{p}^{+}\left(\mathbb{R}^{d}\right), \varphi \in \mathcal{S}\left(\mathbb{R}^{d}\right) .
$$

Using a standard renewal argument (conditioning on the first branching) one verifies that 
Fluctuations of a two-level branching system

$w_{\varphi}$ satisfies the nonlinear integral equation

$$
\begin{aligned}
w_{\varphi}(\mu, t)= & \mathrm{e}^{V_{2} b_{2} t} T_{t}\left(1-\mathrm{e}^{\mathrm{i}\{\cdot \varphi \varphi)}\right)(\mu) \\
& -V_{2} c_{2} \int_{0}^{t} \mathrm{e}^{V_{2} b_{2}(t-s)} T_{t-s}\left(w_{\varphi}(s)^{1+\beta_{2}}\right)(\mu) \mathrm{d} s .
\end{aligned}
$$

The following lemma will be basic:

Lemma 4.4. Let $a_{n}, n=1,2, \ldots$, be a sequence of positive numbers converging to $\infty$. For each $\mu \in \mathcal{M}_{p}^{+}\left(\mathbb{R}^{d}\right), \varphi \in \mathcal{S}\left(\mathbb{R}^{d}\right)$ and $t>0$, let

$$
u_{n}(\mu, t)=a_{n} w_{\varphi a_{n}^{-1}}(\mu, t)
$$

where $w_{\varphi a_{n}^{-1}}$ is defined by (4.11). Then

$$
u_{n}(\mu, t) \rightarrow-\mathrm{i}\left\langle\mu, S_{t} \varphi\right\rangle \mathrm{e}^{\left(V_{1} b_{l}+V_{2} b_{2}\right) t} \quad \text { as } n \rightarrow \infty,
$$

uniformly for $t$ in compact sets.

Proof. By (4.12) $u_{n}$ satisfies

$$
\begin{aligned}
u_{n}(\mu, t)= & \mathrm{e}^{V_{2} b_{2} t} T_{t}\left(a_{n}\left(1-\mathrm{e}^{\mathrm{i}(\cdot, \varphi) a_{n}^{-1}}\right)\right)(\mu) \\
& -V_{2} c_{2} a_{n}^{-\beta_{2}} \int_{0}^{t} \mathrm{e}^{V_{2} b_{2}(t-s)} T_{t-s}\left(u_{n}(s)^{1+\beta_{2}}\right)(\mu) \mathrm{d} s .
\end{aligned}
$$

Hence, by continuous dependence on initial conditions and coefficients (see, for example, Henry 1981), $u_{n}$ converges as $n \rightarrow \infty$, uniformly for $t$ in compact sets, to $u(\mu, t)$ given by

$$
\begin{aligned}
u(\mu, t) & =-\mathrm{ie}^{V_{2} b_{2} t} T_{t}(\langle\cdot, \varphi\rangle)(\mu) \\
& =-\mathrm{i}\left\langle\mu, S_{t} \varphi\right\rangle \mathrm{e}^{\left(V_{1} b_{1}+V_{2} b_{2}\right) t},
\end{aligned}
$$

where we have used (4.4).

Proof of Theorem 3.1 (general case). Since $Y^{n}(0)$ is Poisson with intensity $n R$, we have

$$
\begin{aligned}
\mathrm{E}\left(\exp \left\{\mathrm{in}^{-1}\left\langle\bar{Y}^{n}(t), \varphi\right\rangle\right\}\right) & =\exp \left\{\left\langle\left\langle n R, \mathrm{E} \cdot \mathrm{e}^{\mathrm{i}(X(t), \varphi) n^{-1}}-1\right\rangle\right\rangle\right\} \\
& =\exp \left\{-\left\langle\left\langle R, n w_{\varphi n^{-1}}(t)\right\rangle\right\rangle\right\},
\end{aligned}
$$

where $w_{\varphi n^{-t}}$ is defined by (4.11). Let $u_{n}(\mu, t)=n w_{\varphi n^{-1}}(\mu, t)$. Then, by Lemma 4.4 , we obtain

$$
\lim _{n \rightarrow \infty} \mathrm{E}\left(\exp \left\{\mathrm{in}^{-1}\left\langle\bar{Y}^{n}(t), \varphi\right\rangle\right\}\right)=\exp \left\{\mathrm{i}\left\langle\left\langle R,\left\langle\cdot, S_{t} \varphi\right\rangle \mathrm{e}^{\left(V_{1} b_{1}+V_{2} b_{2}\right) t}\right\rangle\right\rangle\right\}
$$

which implies the desired result.

Proof of Theorem 3.2. Lemma 4.3 gives weak convergence of the one-dimensional distributions of $Z^{n}$. The convergence proof for the finite-dimensional distributions of 
higher order is done by induction on the order. It suffices to do the proof for the twodimensional distributions, since the general argument is analogous.

Let $s<t$ and $\varphi, \psi \in \mathcal{S}\left(\mathbb{R}^{d}\right)$. Conditioning on $\left\{Y^{n}(r), r \leq s\right\}$ and using the Markov property of $\bar{Y}^{n}$ with respect to the filtration of $Y^{n}$ we have

$$
\begin{aligned}
\mathrm{E}\left(\exp \left\{\mathrm{i}\left(\left\langle Z^{n}(s), \varphi\right\rangle+\left\langle Z^{n}(t), \psi\right\rangle\right)\right\}\right)= & \mathrm{E}\left(\exp \left\{\mathrm{i}\left(\left\langle Z^{n}(s), \varphi\right\rangle-\mathrm{E}\left\langle\bar{Y}^{n}(t), \psi\right\rangle n^{-1 / 2}\right)\right\}\right. \\
& \left.\times \mathrm{E}\left[\exp \left\{\mathrm{i}\left\langle\bar{Y}^{n}(t), \psi n^{-1 / 2}\right\rangle\right\} \mid Y^{n}(s)\right]\right) .
\end{aligned}
$$

From (2.6),

$$
\mathrm{E}\left[\exp \left\{\mathrm{i}\left\langle\bar{Y}^{n}(t), \psi n^{-1 / 2}\right\rangle\right\} \mid Y^{n}(s)\right]=\exp \left\{\left\langle\left\langle Y^{n}(s), \log \left(1-w_{\psi n^{-1 / 2}}(\cdot, t-s)\right)\right\rangle\right\rangle\right\},
$$

where $w_{t n^{-1 / 2}}$ is defined by (4.11). Hence

$$
\mathrm{E}\left(\exp \left\{\mathrm{i}\left(\left\langle Z^{n}(s), \varphi\right\rangle+\left\langle Z^{n}(t), \psi\right\rangle\right)\right\}\right)=\exp \left\{B_{n}(s, t)\right\} \mathrm{E}\left(\exp \left\{A_{n}(s, t)\right\}\right),
$$

where

$$
A_{n}(s, t)=\mathrm{i}\left\langle Z^{n}(s), \varphi\right\rangle+\left\langle\left\langle\left(Y^{n}(s)-\mathrm{E}\left(Y^{n}(s)\right)\right) n^{-1 / 2}, n^{1 / 2} \log \left(1-w_{\psi n^{-1 / 2}}(\cdot, t-s)\right)\right\rangle\right\rangle
$$

and

$$
B_{n}(s, t)=-\mathrm{iE}\left(\left\langle\bar{Y}^{n}(t), \psi\right\rangle\right) n^{-1 / 2}+\mathrm{E}\left(\left\langle\left\langle Y^{n}(s), \log \left(1-w_{\psi n^{-1 / 2}}(\cdot, t-s)\right)\right\rangle\right\rangle\right) .
$$

Let

$$
v_{n}(\mu, r)=n^{1 / 2} w_{w^{-1 / 2}}(\mu, r)
$$

By Lemma 4.4,

$$
v_{n}(\mu, r) \rightarrow-\mathrm{i}\left\langle\mu, S_{r} \psi\right\rangle \mathrm{e}^{\left(V_{1} b_{1}+V_{2} b_{2}\right) r} \quad \text { as } n \rightarrow \infty .
$$

We will show that for all $\mu$ and $r$,

$$
n^{1 / 2} \log \left(1-w_{\psi n^{-1 / 2}}(\mu, r)\right) \rightarrow \mathrm{i}\left\langle\mu, S_{r} \psi\right\rangle \mathrm{e}^{\left(V_{1} b_{1}-V_{2} b_{2}\right) r} \quad \text { as } n \rightarrow \infty .
$$

Due to (4.16) and (4.17) it suffices to prove that

$$
\left|n^{1 / 2} \log \left(1-w_{\psi n^{-1 / 2}}(\mu, r)\right)+n^{1 / 2} w_{\psi n^{-1 / 2}}(\mu, r)\right| \rightarrow 0 \quad \text { as } n \rightarrow \infty,
$$

but this follows from the inequality $|\log (1-z)+z| \leq$ const. $|z|^{2}$ for $|z|<\frac{1}{2}$, (4.16) and (4.17). In addition, it is possible to obtain the estimate

$$
\begin{aligned}
& \left|n^{1 / 2} \log \left(1-w_{\psi n^{-1 / 2}}(\mu, r)\right)-\mathrm{i}\left\langle\mu, S_{r} \psi\right\rangle \mathrm{e}^{\left(V_{1} b_{1}+V_{2} b_{2}\right) r}\right| \\
& \quad \leq \text { const. }\left[\mathrm{E}_{\mu}(\langle X(r), \psi\rangle)+n^{-1 / 2} \int_{0}^{r} T_{r-s}\left(\mathrm{E} \cdot\left(\langle X(s), \psi\rangle^{2}\right)\right)(\mu) \mathrm{d} s\right] .
\end{aligned}
$$

Now, since

$$
\begin{aligned}
\left.\mathrm{E}\left(\left\langle\left\langle Y^{n}(s)-\mathrm{E}\left(Y^{n}(s)\right)\right) n^{-1 / 2}, \zeta\right\rangle\right\rangle^{2}\right) & =n^{-1}\left\langle\left\langle n R,(\mathrm{E} \cdot(\zeta(X(s))))^{2}\right\rangle\right\rangle \\
& =\left\langle\left\langle R,(\mathrm{E} \cdot(\zeta(X(s))))^{2}\right\rangle\right\rangle
\end{aligned}
$$


for any $\zeta \in C^{+}\left(\mathcal{M}_{p}^{+}\left(\mathbb{R}^{d}\right)\right)$, we have, by (4.18), (4.19) and the dominated convergence theorem,

$$
\begin{gathered}
\mathrm{E}\left(\left\langle\left\langle\left(Y^{n}(s)-\mathrm{E}\left(Y^{n}(s)\right)\right) n^{-1 / 2}, n^{1 / 2} \log \left(1-w_{\psi n^{-1 / 2}}(\cdot, t-s)\right)\right.\right.\right. \\
\left.\left.\left.-\mathrm{i}\left\langle\cdot, S_{t-s} \psi\right\rangle \mathrm{e}^{\left(V_{1} b_{1}+V_{2} b_{2}\right)(r-s)}\right\rangle\right\rangle^{2}\right) \rightarrow 0 \quad \text { as } n \rightarrow \infty
\end{gathered}
$$

Since

$$
\left.\left\langle\left(\left(Y^{n}(s)-\mathrm{E}\left(Y^{n}(s)\right)\right) n^{-1 / 2},\left\langle\cdot, S_{t-s} \psi\right\rangle\right\rangle\right)=\left\langle Z^{n}(s), S_{t-s} \psi\right\rangle\right\rangle
$$

it follows from (4.15), (4.20) and Lemma 4.3 (convergence of one-dimensional distributions) that

$$
\lim _{n \rightarrow \infty} \mathrm{E}\left(\exp \left\{B_{n}(s, t)\right\}\right)=\exp \left\{-\frac{1}{2}\left\langle\left\langle R, \mathrm{E} .\left(\left\langle X(s), \varphi+S_{t-s} \psi \mathrm{e}^{\left(V_{1} b_{1}+V_{2} b_{2}\right)(t-s)}\right\rangle^{2}\right)\right\rangle\right\rangle\right\}
$$

On the other hand, we have

$$
\lim _{n \rightarrow \infty} A_{n}(s, t)=-\frac{1}{2}\left\langle\left\langle R, \mathrm{E} .\left(\langle X(t), \psi\rangle^{2}\right)-\mathrm{E} .\left(\left\langle X(s), S_{t-s} \psi\right\rangle^{2}\right) \mathrm{e}^{2\left(V_{1} b_{1}+V_{2} b_{2}\right)(t-s\rangle}\right\rangle\right\rangle
$$

which is the statement of Lemma 4.5 below.

The completion of the proof follows from (4.13)-(4.15), (4.21), (4.22), (4.2) and (4.3).

Lemma 4.5. The limit in expression (4.22) holds.

Proof. Put $a \equiv V_{1} b_{1}+V_{2} b_{2}$.

From (2.6), and using (4.11), the multiplicative property of branching systems, and (2.5), we have

$$
\begin{aligned}
\mathrm{E}\left(\left\langle\left\langle Y^{n}\langle s), \log \left(1-w_{\psi n^{-1 / 2}}(\cdot, t-s)\right)\right\}\right\rangle\right) \\
=\left\langle n R, \mathrm{E} \cdot\left(\left\langle\left\langle\sum_{j} \delta_{B_{j}(s)}, \log \mathrm{E} \cdot\left(\exp \left\{\mathrm{i}\left\langle X(t-s), \psi n^{-1 / 2}\right\rangle\right\}\right)\right\rangle\right\rangle\right)\right\rangle \\
=\left\langle\left\langle n R, \mathrm{E} \cdot\left(\sum_{j} \log \mathrm{E}_{B_{j}(s)}\left(\exp \left\{\mathrm{i}\left\langle X(t), \psi n^{-1 / 2}\right\rangle\right\}\right)\right)\right\rangle\right\rangle \\
=\left\langle\left\langle n R, \mathrm{E} \cdot\left(\log \prod_{j} \mathrm{E}_{B_{j}(s)}\left(\exp \left\{\mathrm{i}\left\langle X(t), \psi n^{-1 / 2}\right\rangle\right\}\right)\right)\right\rangle\right\rangle \\
=\left\langle\left\langle n R, \mathrm{E} \cdot\left(\log \mathrm{E}_{\sum_{j} B_{j}(s)}\left(\exp \left\{\mathrm{i}\left(X(t), \psi n^{-1 / 2}\right\rangle\right\}\right)\right)\right\rangle\right\rangle \\
=\left\langle\left\langle n R, \mathrm{E} \cdot\left(\log \mathrm{E}_{X(s)}\left(\exp \left\{\mathrm{i}\left(X(t), \psi n^{-1 / 2}\right\rangle\right\}\right)\right)\right\rangle\right\rangle .
\end{aligned}
$$

Hence $B_{n}(s, t)$ given by (4.15), can be written as $B_{n}(s, t)=\left\langle\left\langle R, \gamma_{n}\right\rangle\right\rangle$, with

$$
\begin{aligned}
\gamma_{n}(\mu)= & \mathrm{E}_{\mu}\left[-\mathrm{i}\left\langle X(s), S_{t-s} \psi\right\rangle \mathrm{e}^{a(t-s)} n^{1 / 2}\right. \\
& \left.+n \log \mathrm{E}_{X(s)}\left(\exp \left\{\mathrm{i}\langle X(t), \psi\rangle n^{-1 / 2}\right\}\right)\right], \quad \mu \in \mathcal{M}_{p}^{+}\left(\mathbb{R}^{d}\right),
\end{aligned}
$$


where we have used (4.1) and (4.10). Therefore it suffices to show that

$$
\gamma_{n}(\mu) \rightarrow-\frac{1}{2}\left[\mathrm{E}_{\mu}\left(\langle X(t), \psi\rangle^{2}\right)-\mathrm{E}_{\mu}\left(\left\langle X(s), S_{t-s} \psi\right\rangle^{2}\right) \mathrm{e}^{2 a(t-s)}\right] \quad \text { as } n \rightarrow \infty,
$$

and then to conclude by the dominated convergence theorem.

From (4.23) we have

$$
\gamma_{n}(\mu)=\mathrm{E}_{\mu}\left[-\mathrm{i}\left\langle X(s), S_{t-s} \psi\right\rangle \mathrm{e}^{a(t-s)} n^{1 / 2}+n \log \left(1-\mathrm{iE}_{X(s)}(\langle X(t), \psi\rangle) n^{-1 / 2}+\theta_{n}(X(s))\right)\right],
$$

where

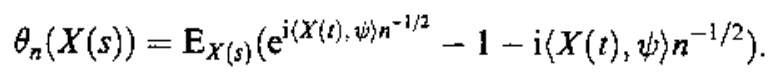

Hence, by (4.1) we have, for large $n$,

$$
\gamma_{n}(\mu)=\mathrm{E}_{\mu}\left\{n \theta_{n}(X(s))-\frac{n}{2}\left[\mathrm{iE}_{X(s)}(\langle X(t), \psi\rangle) n^{-1 / 2}+\theta_{n}(X(s))\right]^{2}+\delta_{n}(X(s))\right\},
$$

where

$$
\delta_{n}(X(s))=n \sum_{k=3}^{\infty} \frac{(-1)^{k-1}}{\bar{k}^{-1}}\left[\mathrm{iE}_{X(s)}(\langle X(t), \psi\rangle) n^{-1 / 2}+\theta_{n}(X(s))\right]^{k}
$$

Now,

$$
\mathrm{iE}_{X(s)}(\langle X(t), \psi\rangle) n^{-1 / 2}+\theta_{n}(X(s))=\mathrm{E}_{X(s)}\left(\mathrm{e}^{\mathrm{j}\langle X(t), \psi\rangle n^{-1 / 2}}-1\right),
$$

and therefore, using (4.1) we have

$$
\begin{aligned}
n \theta_{n}(X(s))- & \frac{n}{2}\left[\mathrm{iE}_{X(s)}(\langle X(t), \psi\rangle) n^{-1 / 2}+\theta_{n}(X(s))\right]^{2} \\
= & n\left(-\frac{1}{2} \mathrm{E}_{X(s)}\left(\langle X(t), \psi\rangle^{2}\right) n^{-1}\right)+\zeta_{n}(X(s)) \\
& -\frac{n}{2}\left[\mathrm{E}_{X(s)}(\langle X(t), \psi\rangle) n^{-1 / 2}\right]^{2}+\eta_{n}(X(s)) \\
= & -\frac{1}{2}\left[\mathrm{E}_{X(s)}\left(\langle X(t), \psi\rangle^{2}\right)-\left\langle X(s), S_{t-s} \psi\right\rangle^{2} \mathrm{e}^{2 a(t-s)}\right] \\
& +\zeta_{n}(X(s))+\eta_{n}(X(s)),
\end{aligned}
$$

where

$$
\zeta_{n}(X(s))=n \mathrm{E}_{X(s)}\left[\mathrm{e}^{\mathrm{i}(X(t), \psi\rangle) n^{-1 / 2}}-1-\mathrm{i}\langle X(t), \psi\rangle n^{-1 / 2}+\frac{1}{2}\langle X(t), \psi\rangle^{2} n^{-1}\right]
$$

and

$$
\eta_{n}(X(s))=-\frac{n}{2}\left\{\left[\mathrm{E}_{X(s)}\left(\mathrm{e}^{\mathrm{i}(X(t), \psi) n^{-1 / 2}}\right)-1\right]^{2}-\left[\mathrm{iE}_{X(s)}(\langle X(t), \psi\rangle) n^{-1 / 2}\right]^{2}\right\}
$$

But

$$
\left|\delta_{n}(X(s))\right|+\left|\zeta_{n}(X(s))\right|+\left|\eta_{n}(X(s))\right| \leq K(X(s)) n^{-1 / 2},
$$

where $K(\cdot)$ is a non-negative function. (The estimate for $\delta_{n}$ uses the inequality 
$\left|\log (1+z)-z+\frac{1}{2} z^{2}\right| \leq|z|^{3}$ for $|z| \leq 2 / 3$. In the estimate for $\eta_{n}$ the key is that, expanding $\mathrm{E}_{X(s)}\left(\exp \left\{\mathrm{i}\langle X(t), \psi\rangle n^{-1 / 2}\right\}\right)$, the term $E_{X(s)}(\langle X(t), \psi\rangle)^{2} n^{-1}$ cancels out.)

On the other hand, the function $K$ satisfies $\langle\langle R, \mathrm{E} .(K(X(s)))\rangle\rangle\left\langle\infty\right.$, because $\beta_{1}=\beta_{2}=1$ implies $\left\langle\left\langle R, E .\left(\langle X(s),|\psi|\rangle^{3}\right)\right\rangle\right\rangle<\infty$.

Hence, from (4.24), (4.25) and (4.26) we have

$$
\gamma_{n}(\mu)=-\frac{1}{2}\left[\mathrm{E}_{\mu}\left(\langle X(t), \psi\rangle^{2}\right)-\mathrm{E}_{\mu}\left(\left\langle X(s), S_{t-s} \psi\right\rangle^{2}\right) \mathrm{e}^{2 a(t-s)}\right]+O\left(n^{-1 / 2}\right) \quad \text { as } n \rightarrow \infty,
$$

and the proof is complete.

Proof of Theorem 3.4. Continuity of the paths can be shown by the criterion of Mitoma (1983a). Using Theorem 3.6 of Bojdecki and Gorostiza (1986) (see also Dawson and Gorostiza 1989), it follows from the form of the covariance functional of $Z$, given by (3.2) and (3.3), that $Z$ is a Markov process and that it satisfies the stated stochastic evolution equation, the bilinear functional $Q_{u}(\varphi, \psi)$ being determined by the formula

$$
Q_{u}(\varphi, \psi)=\frac{\mathrm{d}}{\mathrm{d} u} K_{Z}(u, \varphi ; u, \psi)-K_{Z}(u, \varphi ; u, A \psi)-K_{Z}(u, A \varphi ; u, \psi),
$$

where $K_{Z}(u, \varphi ; u, \psi) \equiv \operatorname{cov}(\langle Z(u), \varphi\rangle,\langle Z(u), \psi\rangle)$ and $A \equiv \Delta_{\alpha}+V_{1} b_{1}+V_{2} b_{2}$.

The distribution of the initial value $Z(0)$ follows from Lemma 4.3 for $t=0$.

The proofs of Theorems 3.8 and 3.10 are based on the following lemma.

Lemma 4.6. Let $b_{1}=b_{2}=0$, and $\beta_{1}=\beta_{2}=1$. For all (non-zero) $\varphi, \psi \in \mathcal{S}\left(\mathbb{R}^{d}\right)$,

$$
\begin{aligned}
\left\langle\left\langle R_{\infty},\langle\cdot, \varphi\rangle\langle\cdot, \varphi\rangle\right\rangle\right\rangle & =\frac{V_{1} c_{1} \Gamma\left(\frac{d-\alpha}{2}\right)}{2^{\alpha} \pi^{d / 2} \Gamma\left(\frac{\alpha}{2}\right)} \int_{\mathbb{R}^{d}} \int_{\mathbb{R}^{d}} \frac{\varphi(x) \psi(y)}{\|x-y\|^{d-\alpha}} \mathrm{d} x \mathrm{~d} y \quad \text { if } d>\alpha, \\
\int_{0}^{\infty}\left\langle\left\langle R_{\infty},\left\langle\cdot, S_{t} \varphi\right\rangle\left\langle\cdot, S_{t} \psi\right\rangle\right\rangle\right\rangle \mathrm{d} t & =\frac{V_{1} c_{1} \Gamma\left(\frac{d-2 \alpha}{2}\right) \Gamma\left(\frac{\alpha}{2}\right)}{2^{2 \alpha-1} \pi^{(d-2) / 2} \Gamma\left(\frac{\alpha+1}{2}\right)} \\
& \times \int_{\mathbb{R}^{d}} \int_{\mathbb{R}^{d}} \frac{\varphi(x) \psi(y)}{\|x-y\|^{d-2 \alpha}} \mathrm{d} x \mathrm{~d} y \quad \text { if } d>2 \alpha, \\
\left\langle\left\langle R_{\infty},\left\langle\cdot, S_{t} \varphi\right\rangle^{2}\right\rangle\right\rangle & =o\left(t^{-1}\right) \text { as } t \rightarrow \infty \quad \text { if } d>\alpha .
\end{aligned}
$$

(The integrals in (4.27) and (4.28) do not exist for $d \leq \alpha$ and $d \leq 2 \alpha$, respectively.)

Proof. The Laplace functional of the equilibrium state $B(\infty)$ for $d>\alpha$ is given by (see Gorostiza and Wakolbinger 1991)

$$
\mathrm{E}(\exp \{-\langle B(\infty), \varphi\rangle\})=\exp \left\{-\langle\lambda, \varphi\rangle+V_{1} c_{1} \int_{0}^{\infty}\left\langle\lambda, u_{\varphi}(t)^{2}\right\rangle \mathrm{d} t\right\}, \quad \varphi \in C_{p}^{+}\left(\mathbb{R}^{d}\right)
$$


where $u_{\varphi}(t)$ solves

$$
u(t)=S_{t} \varphi-V_{1} c_{1} \int_{0}^{t} S_{t-s}\left(u(s)^{2}\right) \mathrm{d} s
$$

On the other hand, the Laplace functional of $B(\infty)$ is given by the canonical representation (see Kallenberg 1983)

$$
\mathrm{E}(\exp \{-\langle B(\infty), \varphi\rangle\})=\exp \left\{-\left\langle\left\langle R_{\infty}, 1-\mathrm{e}^{-\{\cdot, \varphi\rangle}\right\rangle\right\}\right\}, \quad \varphi \in C_{p}^{+}\left(\mathbb{R}^{d}\right) .
$$

By computing

$$
E\left(\langle B(\infty), \varphi\rangle^{2}\right)=\left.\frac{\mathrm{d}^{2}}{\mathrm{~d} u^{2}} \mathrm{E}(\exp \{-\langle B(\infty), \varphi u\rangle\})\right|_{u=0}
$$

from (4.30) (using 4.31)) and also from (4.32) and equating, we obtain

$$
\left\langle\left\langle R_{\infty},(\cdot, \varphi\rangle^{2}\right\rangle\right\rangle=2 V_{1} c_{1} \int_{0}^{\infty}\left\langle\lambda,\left(S_{t} \varphi\right)^{2}\right\rangle \mathrm{d} t
$$

and we note that this result can be extended for $\varphi \in \mathcal{S}\left(\mathbb{R}^{d}\right)$. The value of the integral on the right-hand side of (4.33) can be computed using the Chapman-Kolmogorov equation and the potential of the symmetric $\alpha$-stable process (see Blumenthal and Getoor 1968):

$$
\int_{0}^{\infty}\left\langle\lambda,\left(S_{t} \varphi\right)^{2}\right\rangle \mathrm{d} t=\frac{\Gamma\left(\frac{d-\alpha}{2}\right)}{2^{\alpha+1} \pi^{d / 2} \Gamma\left(\frac{\alpha}{2}\right)} \iint \frac{\varphi(x) \varphi(y)}{\|x-y\|^{d-\alpha}} \mathrm{d} x \mathrm{~d} y \quad \text { if } d>\alpha .
$$

This yields (4.27).

From (4.33) we have

$$
\left.\int_{0}^{\infty}\left\langle\left\langle R_{\infty},\left\langle\cdot, S_{t} \varphi\right\rangle^{2}\right\rangle\right\rangle \mathrm{d} t=2 V_{1} c_{1} \int_{0}^{\infty} \int_{0}^{\infty}\left\langle\lambda, S_{s+t} \varphi\right)^{2}\right\rangle \mathrm{d} s \mathrm{~d} t
$$

The calculation of the last integral reduces to computing $\int_{0}^{\infty} \int_{0}^{\infty} p_{s+r}(x, y) \mathrm{d} s \mathrm{~d} t$, where $p_{t}(x, y)$ is the transition density of the symmetric $\alpha$-stable process (see Iscoe 1986, p. 109). One finds

$$
\int_{0}^{\infty} \int_{0}^{\infty}\left\langle\lambda,\left(S_{s+t} \varphi\right)^{2}\right\rangle \mathrm{d} s \mathrm{~d} t=\frac{\Gamma\left(\frac{d-2 \alpha}{2}\right) \Gamma\left(\frac{\alpha}{2}\right)}{4^{\alpha+1} \pi^{(d-2) / 2} \Gamma\left(\frac{\alpha+1}{2}\right)} \iint \frac{\varphi(x) \varphi(y)}{\|x-y\|^{d-2 \alpha}} \mathrm{d} x \mathrm{~d} y \quad \text { if } d>2 \alpha .
$$

This yields (4.28).

Using the scaling property of the transition function $p_{t}(x)$ of the symmetric $\alpha$-stable process (i.e., $p_{t}(x)=t^{-d / \alpha} p_{1}\left(t^{-1 / \alpha} x\right), t>0, x \in \mathbb{R}^{d}$ ), from (4.27) we have

$$
\begin{aligned}
& t\left\langle\left\langle R_{\infty},\left\langle\cdot, S_{t} \varphi\right\rangle^{2}\right\rangle\right\rangle \\
& \quad=\text { const. } t^{1-2 d / \alpha} \iiint \int \frac{\varphi(z) \varphi(w) p_{1}\left(t^{-1 / \alpha}(z-x)\right) p_{1}\left(t^{-1 / \alpha}(w-y)\right)}{\|x-y\|^{d-\alpha}} \mathrm{d} z \mathrm{~d} w \mathrm{~d} x \mathrm{~d} y
\end{aligned}
$$




$$
=\text { const. } t^{2+d / \alpha} \iiint \int \frac{\varphi\left(t^{1 / \alpha} z\right) \varphi\left(t^{1 / \alpha} w\right) p_{1}(z-x) p_{1}(w-y)}{\|x-y\|^{d-\alpha}} \mathrm{d} z \mathrm{~d} w \mathrm{~d} x \mathrm{~d} y .
$$

Hence

$$
\begin{aligned}
t\left\langle\left\langle R_{\infty},\left\langle\cdot, S_{t} \varphi\right\rangle^{2}\right\rangle\right\rangle \leq & \text { const. } t^{2+d / \alpha-2 n / \alpha}\left(\sup _{t>0, x \in \mathbb{R}^{d}}\left|\varphi\left(t^{1 / \alpha} x\right)\right|^{n / \alpha}(1+\|x\|)^{n}\right)^{2} \\
& \times \iiint \int \frac{p_{\mathrm{l}}(z-x) p_{1}(w-y)}{(1+\|z\|)^{n}(1+\|w\|)^{n}\|x-y\|^{d-\alpha}} \mathrm{d} z \mathrm{~d} w \mathrm{~d} x \mathrm{~d} y,
\end{aligned}
$$

when $n$ is large enough so that the integral is finite. The supremum over $t$ and $x$ is finite because $\varphi \in \mathcal{S}\left(\mathbb{R}^{d}\right)$. Then (4.29) follows by taking $n$ large enough so that $2+d / \alpha-$ $2 n / \alpha<0$.

Proof of Theorem 3.8. Let $R=R_{\infty}$. From (2.3), (3.3), and $S_{t}$-invariance of $\lambda$, we have

$$
\begin{aligned}
\operatorname{var}(\langle Z(t), \varphi\rangle)= & \left(1+2 V_{2} c_{2} t\right)\left\langle\left\langle R_{\infty},\left\langle\cdot, S_{t} \varphi\right\rangle^{2}\right\rangle\right\rangle \\
& \left.+2 V_{1} c_{1} \int_{0}^{t}\left\langle\left\langle R_{\infty},\left\langle\cdot, S_{t-r}\left(S_{r} \varphi\right)^{2}\right)\right\rangle\right\rangle\right\rangle \mathrm{d} r \\
& \left.+4 V_{1} c_{1} V_{2} c_{2} \int_{0}^{t} \int_{0}^{t-r}\left\langle\left\langle R_{\infty},\left\langle\cdot, S_{t-r-v}\left(S_{r+v} \varphi\right)^{2}\right)\right\rangle\right\rangle\right\rangle \mathrm{d} v \mathrm{~d} r \\
= & \left(1+2 V_{2} c_{2} t\right)\left\langle\left\langle R_{\infty},\left\langle\cdot, S_{t} \varphi\right\rangle^{2}\right\rangle\right\rangle \\
& +2 V_{1} c_{1} \int_{0}^{t}\left\langle\lambda,\left(S_{r} \varphi\right)^{2}\right\rangle \mathrm{d} r \\
& +4 V_{1} c_{1} V_{2} c_{2} \int_{0}^{t} \int_{0}^{t-r}\left\langle\lambda,\left(S_{r+v} \varphi\right)^{2}\right\rangle \mathrm{d} v \mathrm{~d} r .
\end{aligned}
$$

The result then follows from the proof of Lemma 4.6. The proof for $R=\delta_{\delta_{x}} \lambda(\mathrm{d} x)$ is similar.

Proof of Theorem 3.10. From (3.4) and (2.3) we have

$$
\mathrm{E}\left\langle\bar{Y}^{n}(\infty), \varphi\right\rangle=\left\langle\left\langle n R_{\infty},\langle\cdot, \varphi\rangle\right\rangle\right\rangle=\langle\lambda, \varphi\rangle n
$$

Hence, using (3.4),

$$
\begin{aligned}
\mathrm{E}\left(\exp \left\{\mathrm{i}\left\langle H^{n}, \varphi\right\rangle\right\}\right)= & \exp \left\{-\mathrm{i}\langle\lambda, \varphi\rangle n^{1 / 2}\right\} \mathrm{E}\left(\exp \left\{\mathrm{i}\left(\bar{Y}^{n}(\infty), \varphi n^{-1 / 2}\right\rangle\right\}\right) \\
= & \exp \left\{-\mathrm{i}\langle\lambda, \varphi\rangle n^{1 / 2}-\left\langle\left\langle R_{\infty}, n\left(1-\mathrm{e}^{\mathrm{i}\langle\cdot \varphi) n^{-1 / 2}}\right)\right\rangle\right\rangle\right. \\
& \left.+V_{2} c_{2} \int_{0}^{\infty}\left\langle\left(R_{\infty},\left(n^{1 / 2} w_{\varphi n^{-1 / 2}}(t)\right)^{2}\right\rangle\right\rangle \mathrm{d} t\right\}
\end{aligned}
$$


But, by (2.3),

$$
-\mathrm{i}\langle\lambda, \varphi\rangle n^{1 / 2}-\left\langle\left\langle R_{\infty}, n\left(1-\mathrm{e}^{\mathrm{i}\langle\cdot, \varphi\rangle n^{-1 / 2}}\right)\right\rangle\right\rangle \rightarrow-\frac{1}{2}\left\langle\left\langle R_{\infty},\langle\cdot, \varphi\rangle^{2}\right\rangle\right\rangle \quad \text { as } n \rightarrow \infty,
$$

and, by Lemma 4.4 ,

$$
n^{1 / 2} w_{\varphi n^{-1 / 2}}(\mu, t) \rightarrow-\mathrm{i}\left\langle\mu, S_{t} \varphi\right\rangle \quad \text { as } n \rightarrow \infty .
$$

Hence $H^{n} \Rightarrow H$ as $n \rightarrow \infty$, and $H$ has characteristic functional

$$
\mathrm{E}(\exp \{\mathrm{i}\langle H, \varphi\rangle\})=\exp \left\{-\frac{1}{2}\left\langle\left\langle R_{\infty},\langle\cdot, \varphi\rangle^{2}\right\rangle\right\rangle-V_{2} c_{2} \int_{0}^{\infty}\left\langle\left\langle R_{\infty},\left\langle\cdot, S_{t} \varphi\right\rangle^{2}\right\rangle\right\rangle \mathrm{d} t\right\} .
$$

The result follows from Lemma 4.6 and Theorem 3.8 .

\section{Further study}

As we have stated, more significant fluctuation limit results on the two-level branching system involve the empirical measure process $Y$ rather than the aggregated process $\bar{Y}$, and this requires a nuclear space of distributions on $\mathcal{M}_{p}^{+}\left(\mathbb{R}^{d}\right)$. To our knowledge no such space has been studied. However, a nuclear space of distributions on the space of probability measures in $\mathbb{R}^{d}$ has been constructed recently by Dawson and Gärtner (1995), and perhaps this construction can be extended to our case. Since $\mathcal{M}_{p}\left(\mathbb{R}^{d}\right) \subset S^{\prime}\left(\mathbb{R}^{d}\right)$, the space of Hida distributions (Hida et al. 1993) might be useful, but it may be too large.

In Remark 3.11 we have already addressed the question of relating the results on the critical dimensions of the two-level superprocess and the two-level fluctuation limit process in the critical case, in particular obtaining results on the former from properties of the latter.

In Remark 3.12 we have raised the question of a relationship between the two-level system and the occupation time of the one-level system in the critical case, which we think should exist.

In Theorem $3.8 R$ is taken to be either $R_{\infty}$ or $\delta_{\delta_{x}} \lambda(\mathrm{d} x)$. On the basis of Theorem 2.2 of Gorostiza and Wakolbinger (1994), it is reasonable to expect that the result should also hold for other elements $R \in \mathcal{M}_{p}^{2+}\left(\mathbb{R}^{d}\right)$ such that $T_{t}^{*} R \rightarrow R_{\infty}$ as $t \rightarrow \infty$.

Fluctuation limits for the cases where $\beta_{1}<1$ and/or $\beta_{2}<1$ lead to non-Gaussian generalized processes. In this case it seems that the results should depend on the relationship between the values of $\beta_{1}$ and $\beta_{2}$.

In addition to the high-density limits studied here, other relevant limit results for the twolevel branching system could be obtained under space-time rescalings (for example, as in Holley and Stroock 1978, for the one-level case).

Another approach to the types of questions studied in this paper would be to start from the two-level branching particle system (without taking the first-level superprocess limit), but it seems this would involve more cumbersome calculations.

From the perspective of applications it may be of interest to prove weak convergence of fluctuations in a Skorokhod space $D\left([0, \infty), S_{q}^{\prime}\left(\mathbb{R}^{d}\right)\right)$, where $S_{q}^{\prime}\left(\mathbb{R}^{d}\right)$ is a Sobolev subspace 
of $\mathcal{S}^{\prime}\left(\mathbb{R}^{d}\right)$, and to find a good value of $q$. It would also be interesting to consider multitype systems with immigration (as in Gorostiza and López Mimbela 1990; Gorostiza and Roelly 1991; Gorostiza et al. 1992; López Mimbela 1992), and interacting branching systems (as in Perkins 1995).

A powerful tool in the theory of superprocesses is the historical process (Dawson and Perkins 1991; Dynkin 1991b; LeGall 1991). It would be of interest to extend the results in this paper to the historical process.

\section{Appendix. Notation and spaces}

$C\left(\mathbb{R}^{d}\right)$ : real continuous functions on $\mathbb{R}^{d}$.

$\varphi_{p}(x)=\left(1+|x|^{2}\right)^{-p}, x \in \mathbb{R}^{d}(p>0)$.

$C_{p}\left(\mathbb{R}^{d}\right)$ : elements $\varphi$ of $C\left(\mathbb{R}^{d}\right)$ such that $\sup _{x}\left|\varphi(x) / \varphi_{p}(x)\right|<\infty$.

$C_{p}^{+}\left(\mathbb{R}^{d}\right)$ : non-negative elements of $C_{p}\left(\mathbb{R}^{d}\right)$.

$\mathcal{S}\left(\mathbb{R}^{d}\right)$ : infinitely differentiable real functions on $\mathbb{R}^{d}$ rapidly decreasing at infinity.

$\mathcal{S}^{\prime}\left(\mathbb{R}^{d}\right)$ : tempered distributions on $\mathbb{R}^{d}$.

$D\left(\left[0, \infty, \mathcal{S}^{\prime}\left(\mathbb{R}^{d}\right)\right)\right.$ : right-continuous with left limits functions from $[0, \infty)$ into $\mathcal{S}^{\prime}\left(\mathbb{R}^{d}\right)$. $\langle\mu, \varphi\rangle=\int_{\mathbb{R}^{d}} \varphi(x) \mu(\mathrm{d} x)$.

$\mathcal{M}_{p}\left(\mathbb{R}^{d}\right)$ : signed Radon measures $\mu$ on $\mathbb{R}^{d}$ such that $\left\langle|\mu|, \varphi_{p}\right\rangle<\infty,(|\mu|=$ variation measure of $\mu$ ).

$\mathcal{M}_{p}^{+}\left(\mathbb{R}^{d}\right)$ : non-negative elements of $\mathcal{M}_{p}\left(\mathbb{R}^{d}\right)$.

$C^{+}\left(\mathcal{M}_{p}^{+}\left(\mathbb{R}^{d}\right)\right)$ : non-negative continuous functions on $\mathcal{M}_{p}^{+}\left(\mathbb{R}^{d}\right)$.

$\langle\langle R, \Phi\rangle\rangle=\int_{\mathcal{M}_{p}^{+}\left(\mathbf{R}^{d}\right)} \Phi(\mu) R(\mathrm{~d} \mu)$.

$\mathcal{M}_{p}^{2+}\left(\mathbb{R}^{d}\right)$ : non-negative Radon measures $\nu$ on $\mathcal{M}_{p}^{+}\left(\mathbb{R}^{d}\right)$ such that $\left\langle\left\langle\nu,\left\langle\cdot, \varphi_{p}\right\rangle\right\rangle\right\rangle<\infty$.

$\lambda$ : Lebesgue measure on $\mathbb{R}^{d}$.

$\delta_{a}$ : Dirac measure at $a$.

$\Rightarrow$ : weak convergence on random elements.

More precise information on some of the spaces above is needed for superprocess limits, but it is not necessary for this paper.

\section{Acknowledgement}

In the writing of this paper the author has benefited from fruitful discussions with Don Dawson and Anton Wakolbinger. Part of this work was done at CIMAT (Guanajuato, Mexico). This research was partially supported by CONACyT grant 211085-5-5-2059E.

\section{References}

Aldous, D. (1989) Stopping times and tightness II. Ann. Probab., 17, 586-595.

Blumenthal, R.M. and Getoor, R.K. (1968) Markov Processes and Potential Theory. New York: Academic Press. 
Bojdecki, T. and Gorostiza, L.G. (1986) Langevin equations for $S^{\prime}$-valued Gaussian processes and fluctuation limits of infinite particle systems. Probab. Theory Related Fields, 73, 227-244.

Bojdecki, T. and Gorostiza, L.G. (1995) Self-intersection local time for Gaussian $\mathcal{S}^{\prime}\left(\mathbb{R}^{d}\right)$-processes: Existence, path continuity and examples. Stochastic Process. Appl., 60, 191-226.

Bojdecki, T. and Jakubowski, J. (1990) Stochastic integration for inhomogeneous Wiener process in the dual of a nuclear space. J. Multivariate Anal., 34, 185-210.

Cremers, H. and Kadelka, D. (1986) On weak convergence of integral functions of stochastic processes with applications to processes taking values in $L_{p}^{E}$. Stochastic Process. Appl., 21, $305-317$.

Dawson, D.A. (1993) Measure-valued Markov processes. In Ecole d'Eté de Probabilités de SaintFlour XXI-1991, pp. 1-260. Lecture Notes in Math. 1541, New York: Springer-Verlag.

Dawson, D.A. and Gärtner, J. (1995) Multilevel large deviations and interacting diffusions II. Preprint.

Dawson, D.A. and Gorostiza, L.G. (1989) Generalized solutions of stochastic evolution equations. In G. Da Prato and L. Tubaro (eds), Stochastic Partial Differential Equations and Applications II, pp. 53-65. Lecture Notes in Math. 1930. New York: Springer-Verlag.

Dawson, D.A. and Gorostiza, L.G. (1990) Generalized solutions of a class of nuclear space-valued stochastic evolution equations. Appl. Math. Optim., 22, 241-263.

Dawson, D.A. and Hochberg, K.J. (1991) A multilevel branching model. Adv. Appl. Probab., 23, 701715 .

Dawson, D.A. and Perkins, E.A. (1991) Historical processes. Mem. Amer. Math. Soc. 454. Providence, RI: American Mathernatical Society.

Dawson, D.A. and Wu, Y. (1995) Multilevel multitype branching models of an information system. In K. Athreya and P. Jagers (eds), Proceedings of IMA Workshop on Branching Processes. To appear.

Dawson, D.A., Hochberg, K.J. and Wu, Y. (1990) Multilevel branching systems. In T. Hida, H.H. Kuo, J. Potthof and L. Streit (eds), White Noise Analysis: Mathematics and Applications, pp. 93107. Singapore: World Scientific.

Dawson, D.A., Hochberg, K.J. and Vinogradov, V. (1994) On path properties of super-2 processes I. In D.A. Dawson (ed.), Measure-valued Processes, Stochastic Partial Differential Equations, and Interacting Systems, pp. 69-82. CRM Proc. Lecture Notes 5. Providence, RI: American Mathematical Society.

Dawson, D.A., Hochberg, K.J. and Vinogradov, V. (1995a) On path properties of super-2 processes II. In M.G. Cranston and M. Pinsky (eds), Proceedings of Symposia in Pure and Applied Mathematics, Series 57, pp. 385-403. Providence, Ri: American Mathematical Society.

Dawson, D.A., Hochberg, K.J. and Vinogradov, V. (1995b) High-density limits of hierarchically structured branching-diffusing populations. Preprint.

Dynkin, B. (1991a) Branching particle systems and superprocesses. Ann. Probab., 19, 1157-1194.

Dynkin, B. (199lb) Path processes and historical super-processes. Probab. Theory Related Fields, 90 , $89-115$.

Etheridge, A. (1993) Limiting behaviour of two-level measure branching. Adv. Appl. Probab., 25, $773-782$.

Fernaindez, B. and Gorostiza, L.G. (1990) Hydrodynamic and fluctuation limits of branching particle systems with changes of mass. Bol. Soc. Mat. Mexicana, 35, 25-37.

Fernández, B. and Gorostiza, L.G. (1992) Convergence of generalized semimartingales to a continuous process. In G. Da Prato and L. Tubaro (eds), Stochastic Partial Differential Equations and Applications, pp. 158-164. Pitman Res. Notes Math. Ser. 268. Harlow: Longman. 
Gorostiza, L.G. (1983) High density limit theorems for infinite systems of unscaled branching Brownian motions. Ann. Probab., 11, 374-392.

Gorostiza, L.G. (1995) Fluctuations of a two-level critical branching system. In A. Etheridge (ed.), Stochastic Partial Differential Equations, Edinburgh 1994. London Math. Soc. Lecture Note Ser. 216, pp. 120-125. Cambridge: Cambridge University Press.

Gorostiza, L.G., Hochberg, K.J. and Wakolbinger, A. (1995) Persistence of a critical super-2 process. J. Appl. Probab., 32, 534-540.

Gorostiza, L.G. and López-Mimbela, J.A. (1990) The multitype measure branching process. Adv. Appl. Probab., 22, 49-67.

Gorostiza, L.G. and López Mimbela, J.A. (1993) A convergence criterion for measure-valued processes, and applications to continuous superprocesses. In D. Nualart and M. Sanz Solé (eds), Barcelona Seminar on Stochastic Analysis, 1992, pp. 62-72. Progr. Probab. 32. Boston: Birkhäuser.

Gorostiza, L.G. and Roelly, S. (1991) Some properties of the multitype measure branching process. Stochastic Process. Appl., 37, 259-274.

Gorostiza, L.G., Roelly, S. and Wakolbinger, A. (1992) Persistence of critical multitype particle and measure branching processes. Probab. Theory Related Fields, 92, 313-335.

Gorostiza, L.G. and Wakolbinger, A. (1991) Persistence criteria for a class of critical branching particle systems in continuous time. Ann. Probab., 19, 266-288.

Gorostiza, L.G. and Wakolbinger, A. (1994) Long time behavior of critical branching particle systems and applications. In D.A. Dawson (ed.), Measure-Valued Process, Stochastic Partial Differential Equations and Interacting Systems, pp. 119-137. CRM Proc. Lecture Notes 5. Providence, RI: American Mathematical Society.

Greven, A. (1991) A phase transition for the coupled branching process I. Probab. Theory Related Fields, 87, 417-458.

Henry, D. (1981) Geometric Theory of Semilinear Parabolic Equations. Lecture Notes in Math. 840. New York: Springer-Verlag.

Hida, T., Kuo, H.-H., Potthoff, J. and Streit, L. (1993) White Noise. An Infinite Dimensional Calculus. Dordrecht: Kluwer.

Hochberg, K.J. (1995) Hierarchically structured branching populations with spatial motion. Rocky Mountain J. Math., 25, 269-283.

Kochberg, K.J. and Wakolbinger, A. (1995) Non-persistence of two-level branching particle systems in low dimensions. In A. Etheridge (ed.), Stochastic Partial Differential Equations, Edinburgh 1994. London Math. Soc. Lecture Note Ser. 216, pp. 126-140. Cambridge: Cambridge University Press.

Holley, R. and Stroock, D.W. (1978) Generalized Ornstein-Uhlenbeck processes and infinite particle branching Brownian motion. Publ. Res. Inst. Math. Sci., Kyoto Univ., 14, 741-788.

Iscoe, 1. (1986) A weighted occupation time for a class of measure-valued branching processes. Probab. Theory Related Fields, 71, 85-116.

Kallenberg, O. (1983) Random Measures. London: Academic Press.

Le Gall, J.F. (1991) A class of path-valued Markov processes and its application to superprocesses. Probab. Theory Related Fields, 95, 25-46.

López-Mimbela, J.A. (1992) Fluctuation limits of multitype branching random fields. J. Multivariate Anal., 40, 56-83.

Mitoma, I. (1983a) On the sample continuity of $S^{\prime}$-process. J. Math. Soc. Japan, 35, 629-636.

Mitoma, I. (1983b) Tightness of probabilities in $C\left([0,1], S^{\prime}\right)$ and $D\left([0,1], S^{\prime}\right)$. Ann. Probab., 11, 989999. 
Perkins, E. (1995) On the Martingale Problem for Interactive Measure-Valued Branching Diffusions. Mem. Amer. Math. Soc. 549. Providence, RI: American Mathematical Society.

Stöckl, A. and Wakolbinger, A. (1994) On clan recurrence and transience in time stationary branching Brownian particle systems. In D.A. Dawson (ed.), Measure-Valued Processes, Stochastic Partial Differential Equations, and Interacting Systems. CRM Proc. Lecture Notes 5, pp. 213-219. Providence, RI: American Mathematical Society.

Treves, F. (1967) Topological Vector Spaces, Distributions and Kernels. New York: Academic Press. Wu, Y. (1991) Dynamic particle systems and multilevel measure branching processes. Ph.D. thesis, Carleton University, Ottawa.

Wu, Y. (1993) Multilevel birth and death particle systern and its continuous diffusion. Adv. Appl. Probab., 25, 549-569.

Wu, Y. (1994a) Asymptotic behavior of two level measure branching processes. Ann. Probab., 22, 854-874.

Wu, Y. (1994b) A three level particle system and existence of general multilevel measure-valued processes. In D.A. Dawson (ed.), Measure-Valued Processes, Stochastic Partial Differential Equations, and Interacting Systems. CRM Proc. Lecture Notes 5, pp. 233-241. Providence, RI: American Mathematical Society.

Received November 1994 and revised December 1995 